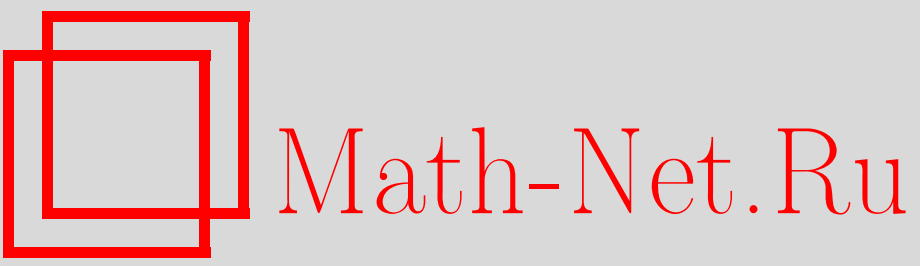

С. В. Клепикова, О. П. Хромова, О предписанных значениях оператора секционной кривизны на трехмерных локально однородных лоренцевых многообразиях, Итоги науки и техн. Сер. Соврем. мат. и ее прил. Темат. обз., 2020, том $180,41-49$

DOI: https://doi.org/10.36535/0233-6723-2020-180-41-49

Использование Общероссийского математического портала Math-Net.Ru подразумевает, что вы прочитали и согласны с пользовательским соглашением

http: //www.mathnet.ru/rus/agreement

Параметры загрузки:

IP : 54.224 .187 .69

26 апреля 2023 г., 14:43:28 


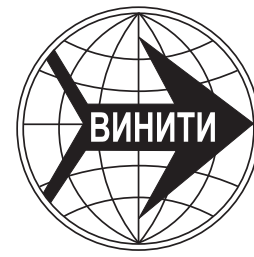

ИТОГИ НАУКИ И ТЕХНИКИ.

Современная математика и ее приложения.

Тематические обзоры.

Том 180 (2020). C. $41-49$

DOI: $10.36535 / 0233-6723-2020-180-41-49$

УДК 514.765

\title{
О ПРЕДПИСАННЫХ ЗНАЧЕНИЯХ \\ ОПЕРАТОРА СЕКЦИОННОЙ КРИВИЗНЫ \\ НА ТРЕХМЕРНЫХ ЛОКАЛЬНО ОДНОРОДНЫХ \\ ЛОРЕНЦЕВЫХ МНОГООБРАЗИЯХ
}

(c) 2020 г. $\quad$ С. В. КЛЕПИКОВА, О. П. ХРОМОВА

\begin{abstract}
АннотАция. В работе решена задача о предписанных значениях оператора секционной кривизны на трехмерном локально однородном лоренцевом многообразии. Получены теоремы о необходимых и достаточных условиях на оператор секционной кривизны такого многообразия.

Ключевые слова: алгебра Ли, группа Ли, левоинвариантная лоренцева метрика, оператор кривизны, спектр.

ON PRESCRIBED VALUES

OF THE OPERATOR OF SECTIONAL CURVATURE ON THREE-DIMENSIONAL LOCALLY HOMOGENEOUS LORENTZIAN MANIFOLDS
\end{abstract}

\author{
(c) 2020 S. V. KLEPIKOVA, O. P. KHROMOVA
}

\begin{abstract}
In this paper, the problem of prescribed values of the operator of sectional curvature on a three-dimensional locally homogeneous Lorentzian manifolds is solved. Necessary and sufficient conditions for the operator of sectional curvature of such manifolds are obtained.
\end{abstract}

Keywords and phrases: Lie algebra, Lie group, left-invariant Lorentzian metric, curvature operator, spectrum.

AMS Subject Classification: 53B20, 53C30, 53C50

1. Введение. Задачи о восстановлении (псевдо)риманова многообразия по предписанному спектру оператора кривизны являются актуальным направлением в исследовании операторов кривизны. Римановы локально однородные пространства с предписанными значениями спектра оператора Риччи были определены О. Ковальским и С. Никчевич в [10]. В случае левоинвариантных лоренцевых метрик на трехмерных группах Ли известна работа Дж. Кальварузо, О. Ковальского [7], в которой исследуется задача о существовании группы Ли с левоинвариантной лоренцевой метрикой и заданными значениями спектра оператора Риччи. Имеется также несколько работ по этой проблеме в неоднородном случае (см. [6,9]).

Подобные результаты были получены Д. Н. Оскорбиным, Е. Д. Родионовым, О. П. Хромовой для оператора одномерной кривизны и оператора секционной кривизны в случае трехмерных

Работа выполнена при поддержке Российского фонда фундаментальных исследований (проект № 18-3100033 мол_а). 
групп Ли с левоинвариантной римановой метрикой (см. [1,2]). Некоторые результаты о восстановлении (псевдо)римановых метрик и аффинных связностей по заданному тензору кривизны содержится в статье Й. Микеша и А. Ванжуровой [12], а также в монографии [11].

Основная цель данной работы - изучить вопрос о предписанных значениях оператора секционной кривизны $\mathcal{K}$ на трехмерных локально однородных лоренцевых многообразиях.

В отличие от случая римановой метрики, где всегда существует ортонормированный базис, в котором матрица оператора $\mathcal{K}$ диагональна, в лоренцевом случае могут возникнуть различные случаи, известные как типь Сегре (см. [4]). Возможны следующие варианты:

1. Тип Сегре $\{111\}: \mathcal{K}$ имеет три действительных собственных значения (возможно, совпадающих), каждому из которых соответствует одномерное собственное подпространство.

2. Тип Сегре $\{1 z \bar{z}\}: \mathcal{K}$ имеет одно действительное и два комплексно сопряженных собственных значения.

3. Тип Сегре $\{21\}: \mathcal{K}$ имеет два действительных собственных значения (возможно, совпадающих), первый из которых имеет алгебраическую кратность 2 , каждому из которых соответствует одномерное собственное подпространство.

4. Тип Сегре $\{3\}: \mathcal{K}$ имеет одно действительное собственное значение алгебраической кратности 3 и соответствующее ему одномерное собственное подпространство.

2. Трехмерные однородные лоренцевы многообразия. Пусть $(M, g)$ - трехмерное однородное многообразие с лоренцевой метрикой $g$ сигнатуры $(+,+,-)$. Обозначим через $\nabla$ связность Леви-Чивиты и через $R$ тензор кривизны, который определим следующим образом:

$$
R(X, Y) Z=\left[\nabla_{Y}, \nabla_{X}\right] Z+\nabla_{[X, Y]} Z .
$$

Лоренцева метрика $g$ индуцирует скалярное произведение $\langle\cdot, \cdot\rangle$ на расслоении $\Lambda^{2} M$ по правилу

$$
\left\langle X_{1} \wedge X_{2}, Y_{1} \wedge Y_{2}\right\rangle=\operatorname{det}\left(\left\langle X_{i}, Y_{i}\right\rangle\right) .
$$

Тензор кривизны $R$ в любой точке можно рассматривать как оператор $\mathcal{K}: \Lambda^{2} M \rightarrow \Lambda^{2} M$, называемый оператором секционной кривизны и определяемый равенством

$$
\langle X \wedge Y, \mathcal{K}(Z \wedge T)\rangle=R(X, Y, Z, T) .
$$

Исследование операторов кривизны на трехмерных локально однородных лоренцевых многообразиях основывается на следующей теореме.

Теорема 1 (Дж. Кальварузо, см. [5]). Пусть $(M, g)$ - трехмерное локально однородное лоренцево многообразие. Тогда либо $(M, g)$ является локально симметричным, либо оно локально изометрично трехмерной группе Ли с левоинвариантной лоренцевой метрикой.

Следующая классификация для трехмерных лоренцевых групп Ли была получена в [3].

Теорема 2. Пусть $G$-трехмерная группа Ли с левоинвариантной лоренцевой метрикой. Справедливы следующие утверждения.

1. Если $G$ унимодулярна, то в алгебре Ли группъ $G$ существует такой псевдо-ортонормированный базис $\left\{e_{1}, e_{2}, e_{3}\right\}$, что метрическая алгебра Ли группы $G$ содержится в следующем списке:

(a) случай $\mathcal{A}_{1}$ :

$$
\left[e_{1}, e_{2}\right]=\lambda_{3} e_{3}, \quad\left[e_{1}, e_{3}\right]=-\lambda_{2} e_{2}, \quad\left[e_{2}, e_{3}\right]=\lambda_{1} e_{1},
$$

где е ер времениподобен;

(b) случай $\mathcal{A}_{2}$ :

$$
\left[e_{1}, e_{2}\right]=\left(1-\lambda_{2}\right) e_{3}-e_{2}, \quad\left[e_{1}, e_{3}\right]=e_{3}-\left(1+\lambda_{2}\right) e_{2}, \quad\left[e_{2}, e_{3}\right]=\lambda_{1} e_{1},
$$

где ез времениподобен;

(c) случай $\mathcal{A}_{3}$ :

$$
\left[e_{1}, e_{2}\right]=e_{1}-\lambda e_{3}, \quad\left[e_{1}, e_{3}\right]=-\lambda e_{2}-e_{1}, \quad\left[e_{2}, e_{3}\right]=\lambda_{1} e_{1}+e_{2}+e_{3},
$$

где ез времениподобен; 
Таблица 1. Трехмерные унимодулярные алгебры Ли

\begin{tabular}{|c|c|c|c|c|}
\hline \multirow{2}{*}{ Алгебра Ли } & \multicolumn{4}{|c|}{ Ограничения на структурные константы } \\
\cline { 2 - 5 } & $\mathcal{A}_{1}$ & $\mathcal{A}_{2}$ & $\mathcal{A}_{3}$ & $\mathcal{A}_{4}$ \\
\hline $\mathrm{su}(2)$ & $(+,+,+)$ & - & - & - \\
\hline $\mathrm{sl}(2, \mathbb{R})$ & $(+,+,-)$ & $\lambda_{1} \neq 0, \lambda_{2} \neq 0$ & $\lambda \neq 0$ & $\lambda_{3} \neq 0$ \\
\hline $\mathrm{e}(2)$ & $(+,+, 0)$ & - & - & - \\
\hline \multirow{2}{*}{$\mathrm{e}(1,1)$} & $(+,-, 0)$ & $\begin{array}{c}\lambda_{1}=0, \lambda_{2} \neq 0 \\
\text { или } \\
\lambda_{1} \neq 0, \lambda_{2}=0\end{array}$ & $\lambda=0$ & $\lambda_{3}=0$ \\
\hline $\mathrm{h}$ & $(+, 0,0)$ & $\lambda_{1}=0, \lambda_{2}=0$ & - & - \\
\hline $\mathbb{R}^{3}$ & $(0,0,0)$ & - & - & - \\
\hline
\end{tabular}

(d) случай $\mathcal{A}_{4}$ :

$$
\left[e_{1}, e_{2}\right]=\lambda_{3} e_{2}, \quad\left[e_{1}, e_{3}\right]=-\beta e_{1}-\alpha e_{2}, \quad\left[e_{2}, e_{3}\right]=-\alpha e_{1}+\beta e_{2},
$$

где е ер времениподобен и $\beta \neq 0$.

2. Если $G$ не является унимодулярной, то в алгебре Ли группы $G$ существует такой псевдоортонормированный базис $\left\{e_{1}, e_{2}, e_{3}\right\}$, что метрическая алгебра Ли группы $G$ содержится в следуюшем списке:

(a) случай $\mathcal{A}$ :

$$
\left[e_{1}, e_{2}\right]=0, \quad\left[e_{1}, e_{3}\right]=\lambda \sin \varphi e_{1}-\mu \cos \varphi e_{2}, \quad\left[e_{2}, e_{3}\right]=\lambda \cos \varphi e_{1}+\mu \sin \varphi e_{2},
$$

где е е времениподобен $u \sin \varphi \neq 0, \lambda+\mu \neq 0, \lambda \geqslant 0, \mu \geqslant 0$;

(a) случай $\mathcal{B}$ :

$$
\left[e_{1}, e_{2}\right]=0, \quad\left[e_{1}, e_{3}\right]=t e_{1}-s e_{2}, \quad\left[e_{2}, e_{3}\right]=p e_{1}+q e_{2},
$$

с ненулевыми $\left\langle e_{2}, e_{2}\right\rangle=-\left\langle e_{1}, e_{3}\right\rangle=1 u q \neq t ;$

(a) случай $\mathcal{C}_{1}$ :

$$
\left[e_{1}, e_{2}\right]=0, \quad\left[e_{1}, e_{3}\right]=s e_{1}+p e_{2}, \quad\left[e_{2}, e_{3}\right]=p e_{1}+q e_{2},
$$

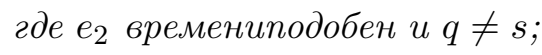

(a) случай $\mathcal{C}_{2}$ :

$$
\left[e_{1}, e_{2}\right]=0, \quad\left[e_{1}, e_{3}\right]=q e_{1}-r e_{2}, \quad\left[e_{2}, e_{3}\right]=p e_{1}+q e_{2},
$$

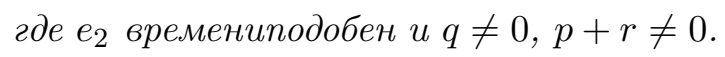

Замечание 1. Существуют ровно шесть неизоморфных трехмерных унимодулярных алгебр Ли и соответствующих им типов унимодулярных трехмерных групп Ли (см. [13]). Все они приведены в таблице 1 вместе с условиями на структурные константы, при которых алгебра Ли имеет данный тип. Если в таблице 1 на пересечении строки, соответствующей алгебре Ли, и столбца, соответствующему типу, стоит знак «-», значит, что для данной алгебры Ли невозможен соответствующий тип базиса. Для случая алгебры Ли $\mathcal{A}_{1}$ указаны только знаки тройки $\left(\lambda_{1}, \lambda_{2}, \lambda_{3}\right)$ с точностью до перестановки и замены знака. Отметим, что подобные базисы также были построены в $[5,8]$.

Следующий классификационный результат для случая трехмерных лоренцевых локально симметричных пространств был получен в [5].

Теорема 3. Трехмерное локально симметричное многообразие $(M, g)$ локально изометрично одному из следующих многообразий:

(I) лоренцевой пространственной форме $\mathbb{R}_{1}^{3}, \mathbb{S}_{1}^{3}$ или $\mathbb{H}_{1}^{3}$ (с нулевой, положстельной или отрицательной секиионной кривизной соответственно);

(II) прямому произведению $\mathbb{R} \times \mathbb{S}_{1}^{2}, \mathbb{R} \times \mathbb{H}_{1}^{2}, \mathbb{S}^{2} \times \mathbb{R}_{1}$ или $\mathbb{H}^{2} \times \mathbb{R}_{1}$; 
(III) многообразию с лоренцевой метрикой $g$, которое допускает такую локальную систему координат $\left(u_{1}, u_{2}, u_{3}\right)$, что метрический тензор имеет вид

$$
g=\left(\begin{array}{ccc}
0 & 0 & 1 \\
0 & \varepsilon & 0 \\
1 & 0 & f\left(u_{2}, u_{3}\right)
\end{array}\right),
$$

где $\varepsilon= \pm 1, f\left(u_{2}, u_{3}\right)=u_{2}^{2} \alpha+u_{2} \beta\left(u_{3}\right)+\xi\left(u_{3}\right), \alpha \in \mathbb{R}, \beta, \xi$-произвольные гладкие фучкиии.

3. Трехмерные лоренцевы группы Ли. Далее под «трехмерной лоренцевой группой Ли $(G, \mathfrak{g}) \gg$ будем понимать трехмерную группу Ли $G$ с левоинвариантной лоренцевой метрикой $g$ и метрической алгеброй Ли $\mathfrak{g}$. Теперь мы готовы доказать следующую теорему.

Теорема 4. Трехмерная унимодулярная лоренцева группа Ли $\left(G, \mathcal{A}_{1}\right)$ с оператором секционной кривизны $\mathcal{K}$ существует тогда и только тогда, когда $\mathcal{K}$ имеет тип Сегре $\{111\}$, а собственные значения $k_{1}, k_{2}, k_{3}$ удовлетворяют одному (или более) из условий:

(i) $k_{1}=k_{2}=k_{3}=0$;

(ii) ровно два из $k_{1}+k_{2}, k_{1}+k_{3} u k_{2}+k_{3}$ равны нулю;

(iii) $\left(k_{1}+k_{2}\right)\left(k_{1}+k_{3}\right)\left(k_{2}+k_{3}\right)<0$.

Доказательство. В рассматриваемом случае матрица оператора секционной кривизны $\mathcal{K}$ диагональна. причем собственные значения $k_{1}, k_{2}, k_{3}$ действительны и равны

$$
\begin{aligned}
& k_{1}=-\frac{1}{4}\left(\lambda_{3}+\lambda_{1}-\lambda_{2}\right)^{2}+\lambda_{1}\left(\lambda_{1}+\lambda_{3}\right), \\
& k_{2}=-\frac{1}{4}\left(\lambda_{1}+\lambda_{2}+\lambda_{3}\right)^{2}+\lambda_{2}\left(\lambda_{1}+\lambda_{2}\right), \\
& k_{3}=-\frac{1}{4}\left(\lambda_{1}+\lambda_{2}-\lambda_{3}\right)^{2}+\lambda_{3}\left(\lambda_{3}-\lambda_{2}\right) .
\end{aligned}
$$

Пусть

$$
\mu_{i}=\frac{1}{2}\left(-\lambda_{1}+\lambda_{2}+\lambda_{3}\right)+\delta_{i} \lambda_{i}
$$

где $\delta_{1}=1, \delta_{2}=\delta_{3}=-1$. Тогда

$$
k_{1}+k_{2}=-2 \mu_{1} \mu_{2}, \quad k_{1}+k_{3}=-2 \mu_{1} \mu_{3}, \quad k_{2}+k_{3}=-2 \mu_{2} \mu_{3} .
$$

Предположим, что как минимум два из $\mu_{i}$ равны нулю. Тогда все три правые части в вышеприведенных уравнениях равны нулю, и система имеет единственное решение $k_{1}=k_{2}=k_{3}=0$.

Пусть один из $\mu_{i}$ равен нулю; тогда ровно два из чисел $k_{1}+k_{2}, k_{1}+k_{3}, k_{2}+k_{3}$ равны нулю.

Предположим, что $\mu_{i} \neq 0$; тогда

$$
\mu_{i}^{2}=-\frac{1}{2} \frac{\left(k_{1}+k_{2}\right)\left(k_{1}+k_{3}\right)\left(k_{2}+k_{3}\right)}{\left(k_{1}+k_{2}+k_{3}-k_{i}\right)^{2}}
$$

и система разрешима тогда и только тогда, когда

$$
\left(k_{1}+k_{2}\right)\left(k_{1}+k_{3}\right)\left(k_{2}+k_{3}\right)<0 .
$$

Теорема 5. Трехмерная унимодулярная лоренцева группа Ли $\left(G, \mathcal{A}_{2}\right)$ с оператором секиионной кривизны $\mathcal{K}$ существует тогда и только тогда, когда выполнено одно из следующих условий:

(i) $\mathcal{K}$ имеет тип Сегре $\{111\}$ с собственными значениями $k_{1}=-k_{2}=-k_{3} \geqslant 0$ с точностью до переобозначения;

(ii) $\mathcal{K}$ имеет тип Сегре $\{12\}$ с собственными значениями
(a) $k_{1}=k_{2}=0$ или
(b) $k_{2}<0$. 
Доказательство. В рассматриваемом случае матрица оператора секционной кривизны $\mathcal{K}$ имеет вид

$$
\mathcal{K}=\left(\begin{array}{ccc}
\frac{3}{4} \lambda_{1}^{2}-\lambda_{1} \lambda_{2} & 0 & 0 \\
0 & 2 \lambda_{2}-\lambda_{1}-\frac{1}{4} \lambda_{1}^{2} & 2 \lambda_{2}-\lambda_{1} \\
0 & -2 \lambda_{2}+\lambda_{1} & -2 \lambda_{2}+\lambda_{1}-\frac{1}{4} \lambda_{1}^{2}
\end{array}\right)
$$

Если $\lambda_{1}=2 \lambda_{2}$, то матрица диагонализируема с собственными значениями $k_{1}=-k_{2}=-k_{3} \geqslant 0$. Иначе, матрица оператора $\mathcal{K}$ имеет следующую жорданову форму:

$$
\mathcal{K}=\left(\begin{array}{ccc}
-\lambda_{1} \lambda_{2}+\frac{3}{4} \lambda_{1}^{2} & 0 & 0 \\
0 & -\frac{1}{4} \lambda_{1}^{2} & 1 \\
0 & 0 & -\frac{1}{4} \lambda_{1}^{2}
\end{array}\right),
$$

и собственные значения равны

$$
k_{1}=-\lambda_{1} \lambda_{2}+\frac{3}{4} \lambda_{1}^{2}, \quad k_{2}=-\frac{1}{4} \lambda_{1}^{2} \leqslant 0 .
$$

Если $k_{2}=0$, то $\lambda_{1}=0$ и все собственные значения равны нулю.

Предположим, что $k_{2}<0$; тогда $\lambda_{1}= \pm 2 \sqrt{-k_{2}}$. Выражая $\lambda_{2}$, получаем

$$
\lambda_{2}=\mp \frac{k_{1}+3 k_{2}}{2 \sqrt{-k_{2}}} \text {. }
$$

Теорема 6. Трехмерная унимодулярная лоренцева группа Ли $\left(G, \mathcal{A}_{3}\right)$ с оператором секиионной кривизны $\mathcal{K}$ существует тогда и только тогда, когда выполнено одно из следующих условий:

(i) $\mathcal{K}$ имеет тип Сегре $\{21\}$ с собственными значениями $k_{1}=k_{2}=0$;

(ii) $\mathcal{K}$ имеет тип Сегре $\{3\}$ с собственным значением $k_{1}<0$.

Доказательство. В данном случае матрица оператора секционной кривизны $\mathcal{K}$ имеет вид

$$
\mathcal{K}=\left(\begin{array}{ccc}
-\frac{1}{4} \lambda^{2} & \lambda & -\lambda \\
\lambda & 2-\frac{1}{4} \lambda^{2} & -2 \\
\lambda & 2 & -2-\frac{1}{4} \lambda^{2}
\end{array}\right)
$$

с совпадающими собственными значениями равными $-\lambda^{2} / 4 \leqslant 0$. Если $\lambda=0$, то $\mathcal{K}$ имеет тип Сегре $\{21\}$ и $k_{1}=k_{2}=0$. Если $\lambda \neq 0$, то $\mathcal{K}$ имеет тип Сегре $\{3\}$ и $k_{1}<0$.

Теорема 7. Трехмерная унимодулярная лоренцева группа Ли $\left(G, \mathcal{A}_{4}\right)$ с оператором секиионной кривизны $\mathcal{K}$ существует тогда и только тогда, когда выполнено одно из следующих условий:

(i) $\mathcal{K}$ имеет muп Сегре $\{111\}$ и $k_{1}=k_{2}=-k_{3}<0$ с точностью до переобозначения;

(ii) $\mathcal{K}$ uмеeт mun Cегре $\{z \bar{z} 1\}$ u $k_{1}+k_{2}<0$.

Доказательство. В данном случае оператор $\mathcal{K}$ имеет собственные значения

$$
k_{1}=\bar{k}_{2}=-\beta^{2}-\frac{1}{4} \lambda_{3}^{2} \pm i \beta\left(2 \alpha-\lambda_{3}\right), \quad k_{3}=-\lambda_{3} \alpha+\frac{3}{4} \lambda_{3}^{2}+\beta^{2},
$$

где $i$ - мнимая единица.

Пусть $x=-\beta^{2}-\lambda_{3}^{2} / 4<0$ и $y=\beta\left(2 \alpha-\lambda_{3}\right)$; тогда $k_{1}=\overline{k_{2}}=x+i y$. Если $\lambda_{3}=2 \alpha$, то

$$
k_{3}=\alpha^{2}+\beta^{2}>0, \quad x=-\alpha^{2}-\beta^{2}<0, \quad y=0 .
$$

Следовательно, $k_{1}=k_{2}=-k_{3}<0$, и собственные значения действительны.

Предположим, что $\lambda_{3} \neq 2 \alpha$; тогда

$$
\beta=\frac{y}{2 \alpha-\lambda_{3}} \neq 0, \quad k_{3}+x=-\lambda_{3} \alpha+\frac{1}{2} \lambda_{3}^{2} .
$$

Если $\lambda_{3}=0$, то

$$
k_{3}=\beta^{2}, \quad x=-\beta^{2}, \quad y=2 \alpha \beta .
$$


Иначе $\lambda_{3} \neq 0$ и $k_{3}+x \neq 0$. Тогда

$$
\alpha=\frac{\lambda_{3}^{2}-2 k_{3}-2 x}{2 \lambda_{3}}, \quad \lambda_{3}= \pm 2\left|k_{3}+x\right| \frac{\sqrt{-x}}{\sqrt{\left(k_{3}+x\right)^{2}+y^{2}}} .
$$

Теорема 8. Трехмернал неунимодулярная лоренцева группа Ли $(G, \mathcal{A})$ с оператором секиионной кривизны $\mathcal{K}$ существует тогда и только тогда, когда $\mathcal{K}$ имеет тип Сегре $\{111\}$, и собственные значения $k_{1}, k_{2}, k_{3}$ удовлетворяют одному (или более) из следующих условий:

(i) $k_{1}=k_{2}=k_{3}>0$;

(ii) $k_{1} k_{3} \leqslant k_{2}^{2}<\left(\frac{k_{1}+k_{3}}{2}\right)^{2}$ и $k_{2}<\frac{k_{1}+k_{3}}{2}$ с точностъю до переобозначения.

Доказательство. В данном случае матрица оператора секционной кривизны $\mathcal{K}$ диагонализируема с действительными собственными значениями $k_{1}, k_{2}, k_{3}$ равными

$$
k_{1}=-\frac{1}{4}(\lambda+\mu)^{2} \cos ^{2} \varphi+\lambda^{2}, \quad k_{2}=-\frac{1}{4}(\lambda+\mu)^{2} \cos ^{2} \varphi+\mu \lambda, \quad k_{3}=-\frac{1}{4}(\lambda+\mu)^{2} \cos ^{2} \varphi+\mu^{2} .
$$

Отметим, что $k_{1}-2 k_{2}+k_{3}=(\lambda-\mu)^{2} \geqslant 0$. Если выполняется равенство нулю, то $\lambda=\mu \neq 0$ и $k_{1}=k_{2}=k_{3}=\lambda^{2} \sin ^{2} \varphi>0$.

Предположим, что $k_{1}-2 k_{2}+k_{3}>0$ и $\lambda \neq \mu$. Рассмотрим разности

$$
k_{1}-k_{2}=\lambda(\lambda-\mu), \quad k_{1}-k_{3}=(\lambda-\mu)(\lambda+\mu) \neq 0, \quad k_{3}-k_{2}=\mu(\mu-\lambda) .
$$

В силу условий $\lambda \geqslant 0, \mu \geqslant 0$ система имеет решение

$$
\lambda=\frac{\left|k_{1}-k_{2}\right|}{\sqrt{k_{1}-2 k_{2}+k_{3}}}, \quad \mu=\frac{\left|k_{2}-k_{3}\right|}{\sqrt{k_{1}-2 k_{2}+k_{3}}} .
$$

Подставляя данное решение назад в (1), получаем

$$
0 \leqslant \cos ^{2} \varphi=4 \frac{k_{2}^{2}-k_{1} k_{3}}{\left(k_{1}-k_{3}\right)^{2}}<1 .
$$

Тогда необходимо $k_{2}^{2}-k_{1} k_{3} \geqslant 0$ и $\left(k_{1}+k_{3}\right)^{2}-4 k_{2}^{2}>0$.

Теорема 9. Трехмерная неунимодулярная лоренцева группа Ли $(G, \mathcal{B})$ с оператором секционной кривизны $\mathcal{K}$ существует тогда и только тогда, когда выполнено одно из следующих условий:

(i) $\mathcal{K}$ имеет muп Сегре $\{111\}$ и $k_{1}=-3 k_{2}=-3 k_{3} \geqslant 0$ с точностью до переобозначения;

(ii) $\mathcal{K}$ uмеeт mun Cегре $\{12\}$ u $k_{1}=-3 k_{2} \geqslant 0$.

Доказательство. В данном случае матрица оператора секционной кривизны $\mathcal{K}$ имеет вид

$$
\mathcal{K}=\left(\begin{array}{ccc}
-\frac{1}{4} s^{2} & q s & p s-q^{2}-q t \\
0 & \frac{3}{4} s^{2} & -q s \\
0 & 0 & -\frac{1}{4} s^{2}
\end{array}\right)
$$

Если $s=q=0$ или $s=0, q=-t$, то матрица оператора $\mathcal{K}$ тривиальна.

Если $p s-q t=0$, то матрица оператора секционной кривизны $\mathcal{K}$ имеет тип Сегре $\{111\}$ с собственными значениями $k_{1}=3 s^{2} / 4, k_{2}=k_{3}=-s^{2} / 4$.

Иначе, матрица оператора $\mathcal{K}$ имеет тип Сегре $\{12\}$ с собственными значениями $k_{1}=3 s^{2} / 4$, $k_{2}=-s^{2} / 4$.

Теорема 10. Трехмерная неунимодулярная лоренцева группа Ли $\left(G, \mathcal{C}_{1}\right)$ с оператором секиионной кривизны $\mathcal{K}$ существует тогда и только тогда, когда выполнено одно из следующих условий:

(i) $\mathcal{K}$ имеет muп Сегре $\{111\}$ с собственными значениями $k_{2} \leqslant 0, k_{3} \leqslant 0, k_{2}^{2}+k_{3}^{2}>0$, $\left|k_{1}\right| \leqslant \sqrt{k_{2} k_{3}}$ с точностью до переобозначения;

(ii) $\mathcal{K}$ имеет muп Сегре $\{1 z \bar{z}\}$, причем $\left|k_{1}\right| \leqslant-\frac{k_{2}+k_{3}}{2}$. 
Доказательство. В данном случае матрица оператора секционной кривизны $\mathcal{K}$ диагонализируема, а ее собственные значения $k_{1}, k_{2}, k_{3}$ равны

$$
\begin{aligned}
& k_{1} 7=q s, \\
& k_{2}=-\frac{1}{2} q^{2}-\frac{1}{2} s^{2}+\frac{1}{2}|q+s| \sqrt{(q-s)^{2}-4 p^{2}}, \\
& k_{3}=-\frac{1}{2} q^{2}-\frac{1}{2} s^{2}-\frac{1}{2}|q+s| \sqrt{(q-s)^{2}-4 p^{2}} .
\end{aligned}
$$

Рассмотрим величину $k_{2} k_{3}-k_{1}^{2}=(q+s)^{2} p^{2} \geqslant 0$. Если выполняется равенство нулю, то

$$
p=0, s= \pm \sqrt{-k_{2}}, q= \pm \sqrt{-k_{3}}, k_{1}=q s \quad \text { или } \quad q=-s, k_{1}=k_{2}=k_{3}=-s^{2}<0 .
$$

В противном случае

Далее рассмотрим систему

$$
k_{2} k_{3}-k_{1}^{2}>0, \quad p^{2}=\frac{k_{2} k_{3}-k_{1}^{2}}{(q+s)^{2}}
$$

имеющую решение

$$
k_{1}=q s, \quad k_{2}+k_{3}=-q^{2}-s^{2}<0,
$$

$$
s= \pm \frac{1}{2} \sqrt{2 \sqrt{\left(k_{2}+k_{3}\right)^{2}-4 k_{1}^{2}}-2\left(k_{2}+k_{3}\right)}, q=\frac{k_{1}}{s}
$$

с необходимым условием $\left(k_{2}+k_{3}\right)^{2}-4 k_{1}^{2} \geqslant 0$.

Отметим, что даже если $k_{2}, k_{3}$ - комплексно сопряженные собственные значения, то $k_{2} k_{3}$ и $k_{2}+$ $k_{3}$ действительны.

Теорема 11. Трехмернал неунимодулярная лоренцева группа Ли $\left(G, \mathcal{C}_{2}\right)$ с оператором секиионной кривизны $\mathcal{K}$ существует тогда и только тогда, когда выполнено одно из следующих условий: (i) $\mathcal{K}$ имеет тип Сегре $\{111\}$ с собственными значениями $\left|\frac{k_{2}+k_{3}}{2}\right|<-k_{1}$ с точностью до
переобозначения;

(ii) $\mathcal{K}$ имеет muп Сегре $\{1 z \bar{z}\}$, причем $\left|\frac{k_{2}+k_{3}}{2}\right|<-k_{1}$.

Доказательство. В данном случае матрица оператора секционной кривизны $\mathcal{K}$ диагонализируема с собственными значениями $k_{1}, k_{2}, k_{3}$ равными

$$
\begin{aligned}
& k_{1}=-q^{2}-\frac{1}{4}(p+r)^{2}<0 \\
& k_{2}=\frac{1}{4}(p+r)^{2}-q^{2}+\frac{1}{2}|p+r| \sqrt{(p-r)^{2}-4 q^{2}} \\
& k_{3}=\frac{1}{4}(p+r)^{2}-q^{2}-\frac{1}{2}|p+r| \sqrt{(p-r)^{2}-4 q^{2}} .
\end{aligned}
$$

Имеем

$$
k_{2}+k_{3}+2 k_{1}=-4 q^{2}<0 \quad \Rightarrow \quad q= \pm \frac{1}{2} \sqrt{-k_{2}-k_{3}-2 k_{1}} .
$$

Пусть $x=p+r \neq 0, y=p-r$. Тогда

$$
x^{2}=k_{2}+k_{3}-2 k_{1}>0, \quad 4 k_{1}^{2}-4 k_{2} k_{3}=y^{2} x^{2} \geqslant 0 .
$$

Данная система имеет решение

$$
x= \pm \sqrt{k_{2}+k_{3}-2 k_{1}}, \quad y= \pm 2 \frac{\sqrt{k_{1}^{2}-k_{2} k_{3}}}{\sqrt{k_{2}+k_{3}-2 k_{1}}} .
$$

Отметим, что даже если $k_{2}, k_{3}$ - комплексно сопряженные собственные значения, то $k_{2} k_{3}$ и $k_{2}+k_{3}$ действительны. 
4. Трехмерные локально симметричные лоренцевы многообразия. Теорема 3 позволяет разделить задачу изучения оператора секционной кривизны трехмерных локально симметричных лоренцевых многообразий на три подзадачи. В то же время, очевидно, что оператор секционной кривизны $\mathcal{K}$ диагонализируем для лоренцевых многообразий постоянной секционной кривизны $\mathbb{R}_{1}^{3}, \mathbb{S}_{1}^{3}$ и $\mathbb{H}_{1}^{3}$ (т.е. $\mathcal{K}$ имеет тип Сегре $\{111\}$ ) и $\mathcal{K}$ имеет три совпадающих собственных значения (равных нулю, положительных или отрицательных соответственно).

В случае прямых произведений (случай (II) теоремы 3 ) оператор секционной кривизны $\mathcal{K}$ имеет тип Сегре $\{111\}$ с двумя равными нулю и третьим ненулевым собственными значениями.

В случае (III) теоремы 3 матрица оператора секционной кривизны имеет вид

$$
\mathcal{K}=\left(\begin{array}{ccc}
0 & 0 & \alpha / \varepsilon \\
0 & 0 & 0 \\
0 & 0 & 0
\end{array}\right)
$$

Тогда либо оператор секционной кривизны $\mathcal{K}$ имеет тип Сегре $\{111\}$ с собственными значениями $k_{1}=k_{2}=k_{3}=0$ для $\alpha=0 ;$ либо $\mathcal{K}$ имеет тип Сегре $\{12\}$ с $k_{1}=k_{2}=0$ для $\alpha \neq 0$. Следовательно, справедлива следующая теорема.

Теорема 12. Трехмерное лоренцево локально симметричное многообразие с оператором секиионной кривизны $\mathcal{K}$ существует тогда и только тогда, когда выполнено одно из следующих условий:

(i) $\mathcal{K}$ имеет тип Сегре $\{111\}$ с равными собственными значениями;

(ii) $\mathcal{K}$ имеет тип Сегре $\{111\}$ с двумя нулевыми и третьим ненулевым собственными значениями;

(iii) $\mathcal{K}$ имеет тип Сегре $\{12\}$ с нулевыми собственными значениями.

5. Оператор секционной кривизны на трехмерных локально однородных лоренцевых многообразиях. В данном разделе, используя результаты предыдущих разделов, мы определим возможный вид оператора секционной кривизны на трехмерных локально однородных лоренцевых многообразиях. Начнем со случая недиагонализируемого оператора секционной кривизны.

Теорема 13. Трехмерное локально однородное лоренцево многообразие $(M, g)$ с недиагонализируемым оператором секционной кривизны $\mathcal{K}$ существует тогда и только тогда, когда $\mathcal{K}$ удовлетворяет одному из следующих условий:

(i) $\mathcal{K}$ uмеeт mun Cегре $\{12\}$ u

(a) оба собственных значения равны нулю или

(b) $k_{2}<0$

(ii) $\mathcal{K}$ имеет тип Сегре $\{3\}$ с отричательным собственным значением;

(iii) $\mathcal{K}$ uмeет mun Cегре $\{1 z \bar{z}\}$ u

(а) комплексно сопряюенные собственные значения имеют отрицательную действительную часть, или

(b) $0 \leqslant \frac{k_{2}+k_{3}}{2}<-k_{1}$.

Доказательство. Тип Сегре $\{12\}$ возможен только для трехмерных лоренцевых групп Ли $\left(G, \mathcal{A}_{2}\right),\left(G, \mathcal{A}_{3}\right)$ or $(G, \mathcal{B})$ и для лоренцева локально симметричного пространства из теоремы 3 (случай (III)). Тогда случай (i) теоремы 13 следует из теорем 5, 6, 9 и 12.

Среди всех вариантов, перечисленных выше, тип Сегре $\{3\}$ возможен только для трехмерной группы Ли $\left(G, \mathcal{A}_{3}\right)$. Тогда из теоремы 6 следует случай (ii) данной теоремы.

Если $\mathcal{K}$ имеет тип Сегре $\{1 z \bar{z}\}$, то $(M, g)$ локально изометрично трехмерной лоренцевой группе Ли $\left(G, \mathcal{A}_{4}\right),\left(G, \mathcal{C}_{1}\right)$ или $\left(G, \mathcal{C}_{2}\right)$. Тогда случай (iii) данной теоремы следует из теорем 7,10 и 11.

Следующая теорема является следствием результатов предыдущих разделов. 
Теорема 14. Трехмерное локально однородное лоренцево многообразие $(M, g)$ с недиагонализируемым оператором секционной кривизны $\mathcal{K}$ с типом Сегре $\{111\}$ существует тогда и толъко тогда, когда собственные значения $k_{1}, k_{2}, k_{3}$ удовлетворяют одному (или более) из следующих условий:

(i) все собственные значения равны друг другу;

(ii) два собственных значения равны нулю, а третье ненулевое;

(iii) ровно два из чисел $k_{1}+k_{2}, k_{1}+k_{3} u k_{2}+k_{3}$ равнъь нулю;

(iv) $\left(k_{1}+k_{2}\right)\left(k_{1}+k_{3}\right)\left(k_{2}+k_{3}\right)<0$;

(v) с точностью до переобозначения

$$
k_{2} k_{3} \leqslant k_{1}^{2}<\left(\frac{k_{2}+k_{3}}{2}\right)^{2} \quad u \quad k_{1}<\frac{k_{2}+k_{3}}{2}
$$

(vi) с точностъю до переобозначения

$$
k_{2}<0, \quad k_{3}<0, \quad\left|k_{1}\right| \leqslant \sqrt{k_{2} k_{3}}
$$

(vii) с точностью до переобозначения

$$
k_{1}<-\left|\frac{k_{2}+k_{3}}{2}\right|
$$

\section{СПИСОК ЛИТЕРАТУРЫ}

1. Гладунова О. П., Оскорбин Д. Н. Применение пакетов символьных вычислений к исследованию спектра оператора кривизны на метрических группах Ли// Изв. АлтГУ. - 2013. - 1-1 (77). - С. 19-23.

2. Оскорбин Д. Н., Родионов Е. Д. О спектре оператора кривизны трехмерных групп Ли с левоинвариантной римановой метрикой// Докл. РАН. - 2013. - 450, № 3. - С. 271-273.

3. Родионов Е. Д., Славский В. В., Чибрикова Л. Н. Локально конформно однородные псевдоримановы пространства// Мат. тр. - 2006. - 9, № 1. - С. 130-168.

4. Bueken P., Djorić M. Three-dimensional Lorentz metrics and curvature homogeneity of order one// Ann. Glob. Anal. Geom. - 2000. - 18. - P. 85-103.

5. Calvaruso G. Homogeneous structures on three-dimensional Lorentzian manifolds// J. Geom. Phys. 2007. - 57. - P. 1279-1291.

6. Calvaruso G. Pseudo-Riemannian 3-manifolds with prescribed distinct constant Ricci eigenvalues// Differ. Geom. Appl. - 2008. - 26. - P. 419-433.

7. Calvaruso G., Kowalski O. On the Ricci operator of locally homogeneous Lorentzian 3-manifolds// Cent. Eur. J. Math. - 2009. - 7, № 1. - P. 124-139.

8. Cordero L. A., Parker P. E. Left-invariant Lorentzian metrics on 3-dimensional Lie groups// Rend. Mat. - 1997. - 17. - P. 129-155.

9. Kowalski O. Nonhomogeneous Riemannian 3-manifolds with distinct constant Ricci eigenvalues// Nagoya Math. J. - 1993. - 132. - P. 1-36.

10. Kowalski O., Nikcevic S. On Ricci eigenvalues of locally homogeneous Riemann 3-manifolds// Geom. Dedic. - 1996. - № 1. - P. 65-72.

11. Mikeš J., Stepanova E., Vanžurová A. et al. Differential Geometry of Special Mappings. — Olomouc: Palacký University, 2015.

12. Mikěs J., Vanžurová A. Reconstruction of an affine connection in generalized Fermi coordinates// Bull. Malays. Math. Sci. Soc. - 2017. - 40, № 1. - P. 205-213.

13. Milnor J. Curvature of left invariant metric on Lie groups// Adv. Math. — 1976. — 21. — P. 293-329.

Клепикова Светлана Владимировна

Алтайский государственный университет, Барнаул

E-mail: klepikova.svetlana.math@gmail.com

Хромова Олеся Павловна

Алтайский государственный университет, Барнаул

E-mail: khromova.olesya@gmail.com 